\title{
Estudio prospectivo de la tecnología en la educación superior en Colombia al 2050
}

\section{Germán Andrés Moreno Cely* Ramón Eduardo Gutiérrez Rodríguez**}

Fecha de recibido: 17 de enero de 2019

Fecha de aprobado: 3 de mayo de 2019

Para citar: Moreno Cely, G. A., Gutiérrez Rodríguez, R. E. (2020). Estudio prospectivo de la tecnología en la educación superior en Colombia al 2050. Universidad E Empresa, 22(38), 160-182. https://doi.org/10.12804/revistas.urosario.edu.co/empresa/a.7583

* Candidato a Magíster en Gestión de Organizaciones, Universidad ecci. Profesional en comercio internacional, Universidad ecci. Técnico profesional en Comercio Exterior y Negocios Internacionales. Correo electrónico: gmorenoc2@ucentral. edu.co,gemoreno@uniandes.edu.co

** Candidato a doctor en Comunicación, Universidad Nacional de La Plata (Argentina). Magíster Université de la Sorbonne Nouvelle, Paris III. Correo electrónico: ramoneduardog@yahoo.com, ramoneduardog@gmail.com 


\section{Resumen}

Esta investigación tiene como eje teórico y conceptual la tecnología y la educación superior en Colombia, plantea como pregunta de investigación ¿Cuáles serán los principales retos y desafíos que tendrá la educación superior en Colombia al año 2050 por los avances tecnológicos? El enfoque metodológico que se utiliza para el desarrollo de esta investigación es de corte mixto cualitativo y cuantitativo, el tipo de investigación es descriptiva y mediante el método del análisis de una encuesta a expertos Delphi, y un taller con expertos. Ellos plantean la necesidad de nuevos modelos de gestión de las universidades hacia una mayor profesionalización de las carreras universitarias, en donde se incluyan estas nuevas tecnologías. Se puede resaltar que las tecnologías de la información y comunicación evidentemente pueden llegar a afectar en su conjunto la educación superior, pero también pueden potenciar y facilitar los procesos de enseñanza y aprendizaje para que sea más incluyente

Palabras clave: tecnología, educación superior, futuro, prospectiva, innovación.

\section{Prospective Study of Technology in Higher Education in Colombia by 2050}

\section{Abstract}

This research has as a theoretical and conceptual axis: technology and higher education in Colombia. The research question was: What will be the main challenges and challenges that higher education in Colombia will have by 2050 due to technological advances? The methodological approach used was a mixed qualitative and quantitative descriptive-type one. The analyzing method was a Delphi expert survey and a workshop with experts. The experts raise the need for new models of management of universities towards a greater professionalization of university careers that include these new technologies. We can emphasize that information and communication technologies can affect higher education as a whole, but they can also strengthen and facilitate teaching and learning processes to make it more inclusive.

Keywords: Technology, university education, future, prospective, innovation.

\section{Estudo prospectivo da tecnologia na educação superior na colômbia ao 2050}

\section{Resumo}

Esta pesquisa tem como eixo teórico e conceitual, a tecnologia e a educação superior na Colômbia, apresenta como pergunta de pesquisa: quais serão os principais desafios que terá a educação superior na Colômbia para o ano 2050 pelos avanços tecnológicos? O Enfoque metodológico que se utiliza para o desenvolvimento desta pesquisa é de corte misto qualitativo e quantitativo, o tipo de pesquisa descritiva e através do método da análise de um inquérito a expertos Delphi, e um workshop com expertos. Os expertos apresentam a necessidade de novos modelos de gestão das universidades a uma maior profissionalização dos cursos universitários onde se incluam estas novas tecnologias. Podemos ressaltar que as tecnologias da informação e comunicação evidentemente podem chegar a afetar em seu conjunto a educação superior, mas também podem potenciar, e facilitar os processos de ensino e aprendizagem para que seja mais inclusiva.

Palavras-chave: tecnologia, educação superior, futuro, prospectiva, inovação. 


\section{Introducción}

En el futuro de la tecnología no existe una posición clara de las diversas disciplinas y campos de conocimiento respecto a si, a diferencia de las revoluciones pasadas, las nuevas tecnologías crearán o destruirán conocimiento en el futuro. Ante esta incertidumbre, son numerosos los gobiernos y organismos que tratan de aproximarse al futuro por medio de la utilización de herramientas de la prospectiva, con el objetivo, en muchos casos, de poder mejorar la orientación de la educación, adaptar y adecuar la planificación de la oferta formativa a las necesidades futuras o mejorar la planificación de políticas sociales, económicas o educativas.

La educación en los últimos años se ha venido transformando de un sistema clásico a un ambiente creativo y dinámico, en el que los estudiantes se están transformando en personas que hacen descubrimientos importantes durante su trabajo individual.

$$
\begin{aligned}
& \text { Múltiples investigaciones demuestran que cuando se hace un uso adecuado del } \\
& \text { software educativo, se pueden alcanzar mejores niveles de aprendizaje. Hoy en día, la } \\
& \text { presencia y facilidad para el uso de medios interactivos de educación, permiten que } \\
& \text { el ser humano aumente sus habilidades (Rodríguez, } 2004 \text { p. 1). }
\end{aligned}
$$

Esta propuesta de investigación tiene como eje teórico y conceptual la tecnología y la educación superior, que son aspectos esenciales en el proyecto de investigación. Además, plantea como pregunta de investigación: ¿cuáles serán los principales retos y desafíos que tendrá la educación superior en Colombia al año 2050 por los avances tecnológicos? En la primera parte del documento, se presenta el marco teórico conceptual, el estado del arte y el marco institucional de la educación en Colombia con sus antecedentes históricos, que tiene como fin contextualizar teóricamente a los lectores. En la segunda parte, se muestra la metodología utilizada para el desarrollo de esta investigación. Posteriormente, en la tercera parte, estarán las fuentes secundarias, iniciando por la ejecución de un taller de expertos y, para recopilar más información, una encuesta que se les realizó, por medio de la aplicación del método Delphi, se identificaron las variables de cambio y los actores que son relevantes para el diseño del plan prospectivo. 


\title{
1. Marcos de referencia
}

Para facilitar la comprensión de la metodología de escenarios, se presenta a continuación un listado de los principales términos utilizados en dicha metodología, con su correspondiente definición. En este marco, se realiza una aproximación conceptual de los temas involucrados en el desarrollo de la investigación, con el fin de poner en contexto a los lectores sobre las definiciones y autores que fundamentan el desarrollo de esta investigación, de esta forma se le da forma y dirección.

La prospectiva aporta teorías, métodos y herramientas útiles para la construcción de un futuro deseado.

\begin{abstract}
Supone movilizar capacidades sociales (técnicas, cognitivas, institucionales) para construir visiones compartidas del porvenir, identificar sus determinantes claves, así como los posibles elementos y factores tanto de ruptura como de continuidad. En breve, requiere disponer de las formas de organización y de acción CEPAL (2011), necesarias para su logro.
\end{abstract}

Es la disciplina que pronostica acciones futuras desde un punto de vista ambiental, social, económico, científico y tecnológico. Uno de los fundadores de la prospectiva la definía como la ciencia que estudia el futuro para comprenderlo y poder influir en él. En esencia, con la prospectiva, se trata de imaginar o proyectar escenarios futuros posibles, condicionados según múltiples variables continuas o discretas, con el fin último de planificar las acciones necesarias para evitar o acelerar su ocurrencia Medina, (2006)

La prospectiva aporta teorías, métodos y herramientas útiles para la construcción de un futuro deseado, la prospectiva se viene aplicando por empresarios preocupados por generar visiones consistentes del futuro del país y de sus organizaciones (Berger, 1964). Uno de los fundadores de la disciplina, la define como "la ciencia que estudia el futuro para comprenderlo y poder influir en él" (Eslava, 2010, p. 5). La prospectiva parte del concepto que el futuro aún no existe y "se puede concebir como una realidad múltiple" (Jouvenel, 1993, p. 20) y que "depende solamente de la acción del hombre" (Godet, 1990, p. 8). Por esa razón, el hombre puede construir el mejor futuro posible, para lo cual debe tomar las decisiones correctas en el momento apropiado (Darcet, 1967). 
En América Latina y el Caribe se describen los logros en la construcción de visionnacionales de desarrollo por los gobiernos de varios países. Según la CEPAL (2013), entre ellos figuran los siguientes ejercicios: Plan Estratégico Industrial 2020 de la Argentina; Brasil 2022; Agenda Chile país desarrollado: más oportunidades y mejores empleos 2018; Visión Colombia 2019; Costa Rica: visión a Largo Plazo 2021; Plan Nacional de Unidad de Guatemala; Estrategia Nacional 2010-2025 del Ecuador; Unidad Nacional de la Esperanza 2032, de Guatemala; Visión de País 2010-2038, de Honduras; Visión Jamaica 2030: Plan Nacional de Desarrollo; Visión Nacional 2030, de México; Paraguay 2015; Plan Perú 2021; Estrategia Nacional de Desarrollo 2030 de la República Dominicana, y Uruguay 2030 (Medina Vásquez, Becerra \& Castaño, 2014).

Lo que se ha denominado la Escuela Francesa sirve para el desarrollo de este proyecto, ya que se sostiene principalmente en el carácter voluntarista de que el futuro se construye desde el presente y no es desde el seguimiento de tendencias que se crean alrededor del elemento de estudio. Adicionalmente, trabaja con la interrelación de variables bajo la concepción de una complejidad de relaciones directas e indirectas que deben ser consideradas, aunque siempre quede latente la incertidumbre propia de la naturaleza de los estudios del futuro.

La escuela francesa (Gaston Berge, Bertrand de Jouvenel, Michel Godet) ha replanteado los términos de la relación conceptual y práctica entre la estrategia y la prospectiva. Esta última permite conjeturar sobre los escenarios futuros y, al hacerlo, proporciona un valioso insumo para definir las líneas estratégicas (plan estratégico) que permiten transitar hacia los escenarios futuros (Zapata, 2006).

\section{Marco institucional de la educación en Colombia}

\subsection{Sistema Educativo Colombiano}

En Colombia la educación se define como un proceso de formación permanente, personal cultural y social que se fundamenta en una concepción integral de la persona 
humana, de su dignidad, de sus derechos y de sus deberes (Ministerio de Educación Nacional, 2009).

En la constitución política colombiana se dan las pautas fundamentales del servicio educativo. "Allí se indica, por ejemplo, que se trata de un derecho de la persona, de un servicio público que tiene una función social y que corresponde al Estado regular y ejercer la suprema inspección y vigilancia respecto del servicio educativo" (Ministerio de Educación Nacional, 2009). La intención es velar por la calidad del servicio, adicionalmente velar por el cumplimiento de los fines y por la mejor formación intelectual y física de los estudiantes. También se establece que se debe garantizar el cubrimiento del servicio educativo con las mejores condiciones y facilidades de acceso.

El sistema educativo colombiano lo conforman la educación inicial, la educación preescolar, la educación básica (primaria cinco grados y secundaria cuatro grados), la educación media (dos grados y culmina con el título de bachiller) y la educación superior.

\subsection{La educación superior}

La educación superior se imparte en dos niveles: pregrado y posgrado. El nivel de pregrado tiene, a su vez, tres niveles de formación:

- Nivel Técnico Profesional (relativo a programas Técnicos Profesionales).

- Nivel Tecnológico (relativo a programas tecnológicos).

- Nivel Profesional (relativo a programas profesionales universitarios).

La educación de posgrado comprende los siguientes niveles:

Especialización

Maestrías

Doctorados

Pueden acceder a los programas formales de pregrado, quienes acrediten el título de bachiller y el Examen de Estado, que es la prueba oficial obligatoria que presentan quienes egresan de la educación media y aspiran a continuar estudios de educación superior (Ministerio de Educación Nacional, 2009). 


\subsection{Antecedentes históricos de la educación superior en Colombia}

"La educación superior en Colombia se inicia en la Colonia, los estudios superiores se crearon teniendo en cuenta la normativa del Código de las Siete Partidas que expidió en 1254 Alfonso X el Sabio" (Guerrero, 2002). La universidad tenía por objetivo la formación de las personas que necesitaba el sector público para sus trabajos civiles (abogados) y en el sector religioso eclesiástico (sacerdotes). Las fundaciones de educación se denominaron de "Estudios Generales que correspondían a las aprobadas por el Rey y el Papa. La Universidad Oficial o Mayor o General estaba sometida al Real Patronato, la Corona intervenía en el gobierno, sus rentas provenían principalmente de la real hacienda" (Guerrero, 2002), tenían una organización muy parecida y tenían de todos los privilegios de las universidades españolas.

Con este propósito,
En 1826 se fundaron las universidades centrales y públicas de Quito, Bogotá y Caracas (Rodríguez \& Burbano, 2012). Después de la disolución de la Gran Colombia, se aprueba una reforma educativa liderada por Mario Ospina Rodríguez que impulsa la educación técnica y científica (Melo, Ramos, \& Hernández (2014, p. 64).

Todo esto limita la libertad de enseñanza e introduce un carácter católico, debido al papel protagónico que tenía la iglesia. Posteriormente, los gobiernos de:
Tomás Cipriano de Mosquera y de José Hilario López, bajo un escenario agitado desde el punto de vista político, adoptan un programa educativo liberal y menos intervencionista. Durante estos años, las medidas estatales sobre educación superior estuvieron marcadas por las diferencias ideológicas entre liberales y conservadores (Melo et al., 2014, p. 64).

Hay que recordar que en 1861 el General Mosquera expulsó a los jesuitas del país y expropió sus bienes a través de lo que se conoció como la "desamortización de bienes de manos muertas" (Melo et al., 2014). 


\subsection{Ministerio de Educación Nacional}

Compete al Ministerio de Educación Nacional, entre otros objetivos, la operación del sistema de aseguramiento de la calidad de la educación superior, la pertinencia de los programas, la evaluación permanente y sistemática, la eficiencia y transparencia de la gestión para facilitar la modernización de las instituciones de educación superior, implementar un modelo administrativo por resultados y la asignación de recursos con su racionalidad.

\subsection{Sistema Nacional de Acreditación en Colombia}

El sistema Nacional de Acreditación (SNA) es el conjunto de políticas, estrategias, procesos y organismos cuyo objetivo fundamental es garantizar a la sociedad que las instituciones de educación superior que hacen parte del sistema cumplen con los más altos requisitos de calidad y que realizan sus propósitos y objetivos (Colombia, 1992).

La Acreditación es un testimonio que da el Estado sobre la calidad de un programa o institución, con base en un proceso previo de evaluación en el cual intervienen la institución, las comunidades académicas y el Consejo Nacional de Acreditación.

Este proceso ha estado muy ligado, desde sus inicios, a la idea de la autonomía y la autorregulación, complementada con la exigencia de rendición de cuentas que se hace a la Educación Superior desde distintos sectores sociales. Por tal motivo, debe subrayarse que la legitimidad del Sistema está fuertemente ligada con los propósitos de la comunidad académica misma y sus interacciones con la sociedad. Dicha legitimidad es el resultado de una larga deliberación en la que se han venido logrando consensos alrededor de los puntos centrales del Sistema: el modelo y sus fases, el concepto de calidad, los factores y características, la metodología para la autoevaluación, la evaluación por pares, etc. La evaluación externa se entiende como un complemento a estos procesos y, por lo tanto, los actos de acreditación expresan fundamentalmente la capacidad de autorregulación de las instituciones (Ministerio de Educación Nacional, 2009). 


\section{Estado del arte}

La revisión bibliográfica objeto del estudio está compuesta por las publicaciones de investigación de estudios prospectivos del impacto de la tecnología en el trabajo. La búsqueda sistemática contempló herramientas como Scopus, Science Direct y ACM Digital Library que suministraron información amplia y completa de publicaciones, magazines y resúmenes de literatura científica.

Los descriptores de búsqueda o conceptos clave utilizados se muestran en la tabla 1.

Tabla 1. Estado del Arte en los últimos años de la prospectiva en el entorno en la Educación Superior

\begin{tabular}{|c|c|c|c|c|c|}
\hline Título & Año & Autor & Variables & Descripción & Conclusiones \\
\hline $\begin{array}{l}50 \text { estrategias } \\
\text { para } 2050 . \text { El } \\
\text { trabajo y la revo- } \\
\text { lución digital en } \\
\text { España }\end{array}$ & 2018 & $\begin{array}{l}\text { Proyecto } \\
\text { Millennium en } \\
\text { colaboración } \\
\text { con Fundación } \\
\text { Telefónica }\end{array}$ & $\begin{array}{l}\text { Empleo, pros- } \\
\text { pectiva, tecnolo- } \\
\text { gía, España, }\end{array}$ & $\begin{array}{l}\text { Analiza los efectos } \\
\text { que en España } \\
\text { pueden tener los } \\
\text { cambios tecnológi- } \\
\text { cos asociados a la } \\
\text { revolución digital, } \\
\text { con especial } \\
\text { atención a los que } \\
\text { afectan al mundo } \\
\text { del trabajo }\end{array}$ & $\begin{array}{l}\text { Las tecnologías de las } \\
\text { últimas décadas han } \\
\text { creado tantos o más } \\
\text { puestos de trabajo y } \\
\text { tipos de empleos que } \\
\text { los que han sustituido. } \\
\text { El concepto de desem- } \\
\text { pleo ha perdido su sig- } \\
\text { nificado para las nuevas } \\
\text { generaciones. }\end{array}$ \\
\hline $\begin{array}{l}\text { Estrategias para } \\
2050 \text { en busca } \\
\text { del mejor esce- } \\
\text { nario posible }\end{array}$ & 2018 & Mercedes Núñez & $\begin{array}{l}\text { Educación, } \\
\text { Tecnología, tra- } \\
\text { bajo, econimía, } \\
\text { politica, prospec- } \\
\text { tiva }\end{array}$ & $\begin{array}{l}\text { El informe es fruto } \\
\text { de una profunda } \\
\text { reflexión sobre el } \\
\text { presente como } \\
\text { la mejor manera } \\
\text { de anticiparse al } \\
\text { futuro. }\end{array}$ & $\begin{array}{l}\text { Apuesta por la ciberse- } \\
\text { guridad, colaboración } \\
\text { hombre-máquina, del } \\
\text { loT al loB, inteligencia } \\
\text { artificial, la diversidad } \\
\text { como oportunidad, la } \\
\text { educación como pilar de } \\
\text { cualquier plan de futuro. }\end{array}$ \\
\hline $\begin{array}{l}\text { Estudio pros- } \\
\text { pectivo sobre la } \\
\text { gestión del co- } \\
\text { nocimiento en la } \\
\text { educación supe- } \\
\text { rior en Colombia } \\
\text { al año } 2025\end{array}$ & 2017 & $\begin{array}{l}\text { MAGERLY } \\
\text { ALEJANDRA } \\
\text { CARDOZO } \\
\text { SUÁREZ } \\
\text { RICARDO CAÑON } \\
\text { MORENO } \\
\text { OSCAR EDUARDO } \\
\text { CAMELO } \\
\text { NOHORA MILENA } \\
\text { HERNANDEZ } \\
\text { JAVIER PUENTES } \\
\text { VELASCO }\end{array}$ & $\begin{array}{l}\text { Calidad, } \\
\text { Educación supe- } \\
\text { rior, Gestión del } \\
\text { conocimiento, } \\
\text { Prospectiva estra- } \\
\text { tégica, variables, } \\
\text { actores. }\end{array}$ & $\begin{array}{l}\text { Se aplicó una } \\
\text { encuesta con el } \\
\text { fin de conocer la } \\
\text { percepción de los } \\
\text { expertos frente a } \\
\text { la problemática } \\
\text { presentada y para } \\
\text { plantear diversas } \\
\text { estrategias que } \\
\text { contribuyan a su } \\
\text { solució }\end{array}$ & $\begin{array}{l}\text { Se identificaron las va- } \\
\text { riables que son impor- } \\
\text { tantes, así como la defi- } \\
\text { nición de los diferentes } \\
\text { actores junto con sus } \\
\text { objetivos, que son rele- } \\
\text { vantes para emprender } \\
\text { la construcción del plan } \\
\text { prospectivo de los retos } \\
\text { y desafíos de la gestión } \\
\text { del conocimiento en la } \\
\text { educación superior en } \\
\text { Colombia. }\end{array}$ \\
\hline
\end{tabular}

Continúa 


\begin{tabular}{|c|c|c|c|c|c|}
\hline Título & Año & Autor & Variables & Descripción & Conclusiones \\
\hline $\begin{array}{l}\text { La Educación } \\
\text { Superior Inclusiva } \\
\text { en Algunos } \\
\text { Países de } \\
\text { Latinoamérica: } \\
\text { Avances, } \\
\text { Obstáculos y } \\
\text { Retos }\end{array}$ & 2017 & M. Stella Fajardo & $\begin{array}{l}\text { Derecho a la edu- } \\
\text { cación, Política } \\
\text { Educacional, } \\
\text { Práctica pedagó- } \\
\text { gica, Universidad, } \\
\text { Diversidad } \\
\text { cultural. }\end{array}$ & $\begin{array}{l}\text { La base del análisis } \\
\text { es de carácter do- } \\
\text { cumental, se con- } \\
\text { sultaron artículos, } \\
\text { tesis de grado e } \\
\text { informes, que per- } \\
\text { mitieron evidenciar } \\
\text { la evolución de las } \\
\text { Universidades en el } \\
\text { tránsito del sistema } \\
\text { educativo hacia } \\
\text { una educación } \\
\text { inclusiva para las } \\
\text { personas con dis- } \\
\text { capacidad. }\end{array}$ & $\begin{array}{l}\text { Las políticas públicas y } \\
\text { lineamientos institucio- } \\
\text { nales para el acceso a } \\
\text { la educación superior } \\
\text { de la población con } \\
\text { discapacidad es posible } \\
\text { afirmar que si bien, } \\
\text { existe un vasto soporte } \\
\text { constitucional y legal en } \\
\text { la mayoría de los países } \\
\text { iberoamericanos, sobre } \\
\text { los derechos de las per- } \\
\text { sonas con discapacidad. }\end{array}$ \\
\hline $\begin{array}{l}\text { Los desafíos de } \\
\text { la gobernanza } \\
\text { de la educación } \\
\text { superior en } \\
\text { lberoamérica }\end{array}$ & 2017 & $\begin{array}{l}\text { Dr. J.J. Brunner } \\
\text { Dra. Liliana } \\
\text { Pedraja-Reja }\end{array}$ & $\begin{array}{l}\text { Educacíon, } \\
\text { Desafios, } \\
\text { Iberoamerica }\end{array}$ & $\begin{array}{l}\text { Un reciente } \\
\text { informe sobre la } \\
\text { educación superior } \\
\text { en lberoamérica } \\
\text { muestra el cam- } \\
\text { biante panorama y } \\
\text { la velocidad con la } \\
\text { cual está transfor- } \\
\text { mándose este sec- } \\
\text { tor en la mayoría } \\
\text { de los países de la } \\
\text { región. }\end{array}$ & $\begin{array}{l}\text { En el futuro próximo, } \\
\text { la gobernanza de los } \\
\text { sistemas deberá asumir } \\
\text { un orden adicional de } \\
\text { desafíos, relacionado } \\
\text { con la imprescindible } \\
\text { innovación de las } \\
\text { funciones propiamente } \\
\text { académicas: docencia, } \\
\text { investigación, vincula- } \\
\text { ción con la sociedad } \\
\text { civil y participación en la } \\
\text { esfera pública. }\end{array}$ \\
\hline
\end{tabular}

Fuente: elaboración propia.

En este análisis documental se recogieron las principales tendencias a largo plazo y los factores de cambio relevantes en la educación superior, "las tecnologías futuras pueden reemplazar gran parte de los trabajos desarrollados por el ser humano" (Fundación Telefonica, 2018, p. 14), las opiniones apuntan a que este mayor avance tecnológico podría conducir a nuevas oportunidades de trabajo y educación, incluso a un incremento mundial de la investigación que beneficie a los seres humanos. "Es relevante la especial importancia que los expertos conceden al aprendizaje y al desarrollo de competencias digitales, dentro de las estrategias clave para afrontar los desafíos que se nos presentan" (Núñez, 2018). La educación aparece como uno de los pilares de cualquier plan de futuro y como una tarea en la que deben participar todos los agentes sociales. En este momento, la educación está avanzando hacia una inclusión y el desarrollo que permita fortalecer las capacidades de las personas para afrontar las nuevas tecnologías, "Se reconoce que aunque las Tic se constituyen 
en una herramienta efectiva para la inclusión educativa, no alcanzan la cobertura requerida y la mayoría de la población con discapacidad no tiene acceso aún a ellas" (Fajardo, 2017). Una educación superior con nuevas tecnologías puede estar garantizando el ingreso en diferentes campos educativos a personas que no han tenido oportunidad de adquirirla de forma permanente, lo que generaría oportunidades laborales.

La educación en Colombia se enfrenta a varios retos, es necesario "que cada institución posea currículos pedagógicos en donde se dé prioridad a la gestión del conocimiento y se acoplen a las necesidades que presenta la sociedad" (Suárez, Moreno, Camelo, Hénández \& Velaso, 2017, p. 41), ya que debe ser capaz de reinventarse y cambiar para afrontar los desafíos futuros en términos de los valores cambiantes de la sociedad, el uso de tecnologías en el aprendizaje, la formación para profesiones desconocidas, nuevos paradigmas educativos, y un mundo más complejo y global.

En el futuro próximo, la gobernanza de los sistemas deberá asumir un orden adicional de desafíos, relacionado con la imprescindible innovación de las funciones propiamente académicas: docencia, investigación, vinculación con la sociedad civil y participación en la esfera pública (Fajardo, 2017, p. 24).

La tendencia es que la estrategia educativa que se adopte hoy será determinante para que se puedan afrontar con garantías los cambios tecnológicos que se tienen por delante.

\section{Metodología utilizada en la investigación}

Para el desarrollo del Estudio Prospectivo de la Tecnología en la Educación Superior en Colombia al 2050, se emplea una metodología descriptiva que abarca las estrategias mencionadas en la figura 1. 
Figura 1. Metodología desarrollada en el Estudio Prospectivo de la Tecnología en la Educación Superior en Colombia al 2050

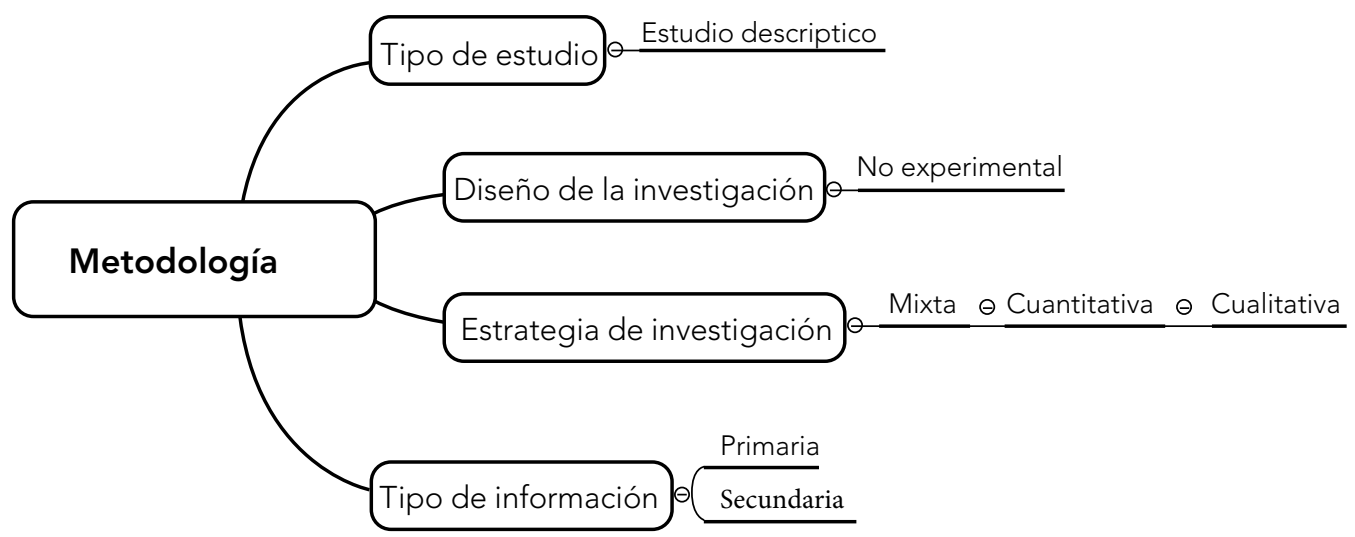

Fuente: elaboración propia.

Para el desarrollo de este proyecto de investigación se utilizó el modelo de la escuela francesa llamado etapas fundamentales de la prospectiva estratégica, "no obstante que se trata de un modelo elemental, es necesario tener claridad hacia dónde va el tema que estamos analizando. Es decir, cuáles son las tendencias mundiales del negocio, si se trata del análisis prospectivo" (Mojica, 2008), ya que permite tomar varios futuros posibles y da una visión más amplia sobre las mejores estrategias que pueden aplicar en esta investigación, para lograrlo se aplicará el modelo básico en donde se explican las etapas fundamentales para lograr un buen estudio prospectivo, estas etapas se muestran en la figura 2.

Figura 2 Dos Modelos de Prospectiva Estratégica

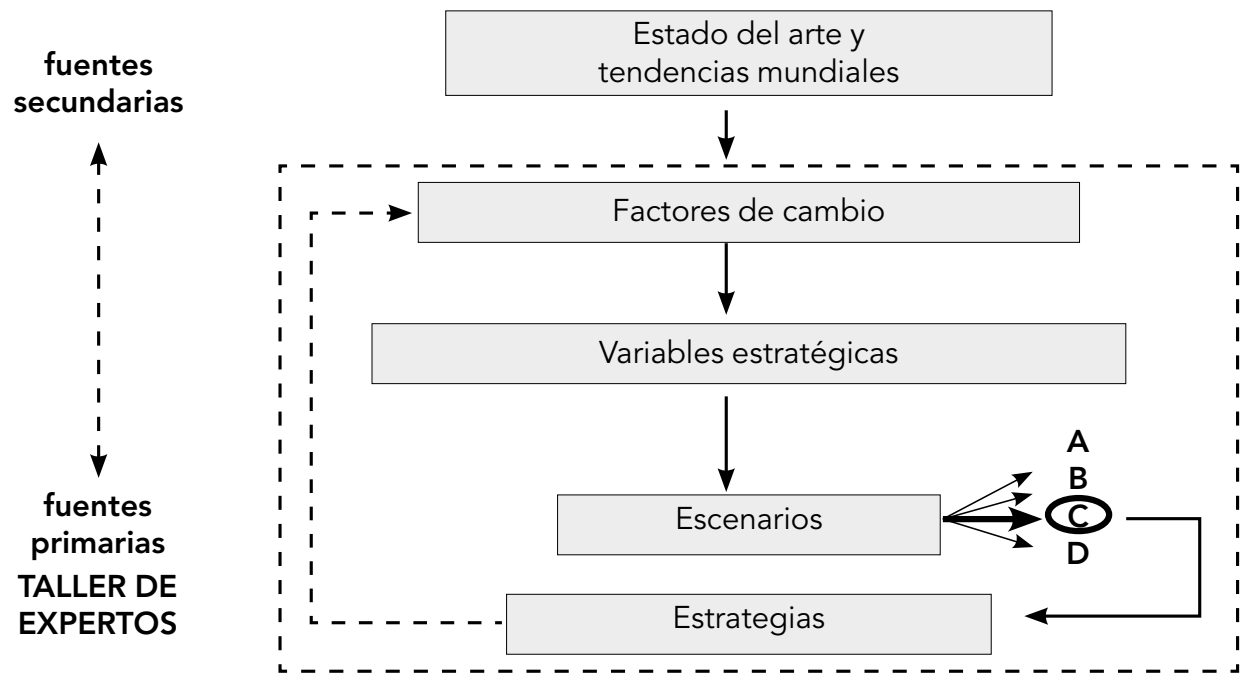

Fuente: Mojica (2008) 
El enfoque metodológico que se utilizará para el desarrollo de esta investigación es de corte mixto cualitativo y cuantitativo, el tipo de investigación descriptiva y mediante el método del análisis de una encuesta a expertos Delphi.

La prospectiva francesa sirve para el desarrollo de este proyecto, ya que se sostiene principalmente en el carácter voluntarista de que el futuro se construye desde el presente y no es desde el seguimiento de tendencias que se crean alrededor del elemento de estudio. Adicionalmente, trabaja con la interrelación de variables bajo la concepción de una complejidad de relaciones directas e indirectas que deben ser consideradas, aunque siempre quede latente la incertidumbre propia de la naturaleza de los estudios del futuro.

Teniendo en cuenta los planteamientos de la Escuela Francesa y específicamente la corriente Voluntarista y la metodología diseñada por el The Millennium Project, se tomó la decisión de realizar el estudio prospectivo.

El proyecto Millennium se trata de un Think Tank, una red de pensamiento, creada hace ya 20 años, cuyo objetivo es realizar una investigación global sobre futuros globales. Esta investigación trabaja y se desarrolla por medio de una amplia participación canalizada por los cerca de 60 nodos repartidos por todo el mundo. Por medio de estos nodos participan en la reflexión sobre el futuro más de 2500 personas expertas en diferentes ámbitos de conocimiento, perspectivistas. La red tiene un carácter transinstitucional; los participantes trabajan para las Naciones Unidas, gobiernos, universidades, fundaciones, ong, institutos de investigación, de más de 60 países y se financia por una amplitud de instituciones.

\section{Resultados de la investigación}

Esta encuesta Delphi fue realizada con expertos procedentes de diversos ámbitos de competencia y conocimiento (universidades, empresas, centros de ciencia y tecnología, especialistas sectoriales de la economía del sector público y privado), para_tener una aproximación al escenario más positivo. En este sentido, el objetivo de la encuesta no ha sido otro que el de definir un marco de referencia y de reflexión, a fin de construir las conclusiones de esta investigación. Se trata de ajustar el análisis a los condicionantes 
demográficos, socioeducativos, económicos, políticos y tecnológicos, para comenzar a esbozar cuáles serían los factores clave y las potenciales estrategias con mayor impacto sobre la construcción de futuro de la educación superior en Colombia.

En la tabla 2 se puede ver el perfil de los 198 expertos que participaron en la encuesta Delphi elaborada para el presente estudio.

Tabla 2. Demografía de la encuesta desarrollada en el trabajo prospectivo

\begin{tabular}{lc}
\hline \multicolumn{3}{c}{ Demografía } \\
\hline \multicolumn{2}{c}{ Género } \\
\hline \multicolumn{2}{c}{ Residentes en Colombia } \\
\hline Hombres & $37 \%$ \\
\hline Mujeres & $61 \%$ \\
\hline \multicolumn{2}{c}{ Formación Profesional } \\
\hline Posdoctoral & $11 \%$ \\
\hline Doctoral & $8 \%$ \\
\hline Maestría & $22 \%$ \\
\hline Especialización & $27 \%$ \\
\hline Pregrado & $32 \%$ \\
\hline & Experiencia en educación \\
\hline Alta & $5 \%$ \\
\hline Media & $8 \%$ \\
\hline Baja & $2 \%$ \\
\hline
\end{tabular}

Fuente: elaboración propia.

\subsection{Factores}

A continuación, se muestran las valoraciones de diferentes preguntas. La evaluación se hace entre 1 y 4 , siendo 4 el más alto.

La figura 3 muestra que hay consenso en no centrarse solo en anticipar si habrá cambios en las profesiones en esta era de cambios tecnológicos cuando se preguntó: ¿Debido al avance científico y tecnológico de la Cuarta Revolución Industrial, la mayor parte de las profesiones actuales serán obsoletas en el 2050? 
Figura 3. Respuestas de expertos sobre los avances científicos y tecnológicos de la Cuarta Revolución Industrial

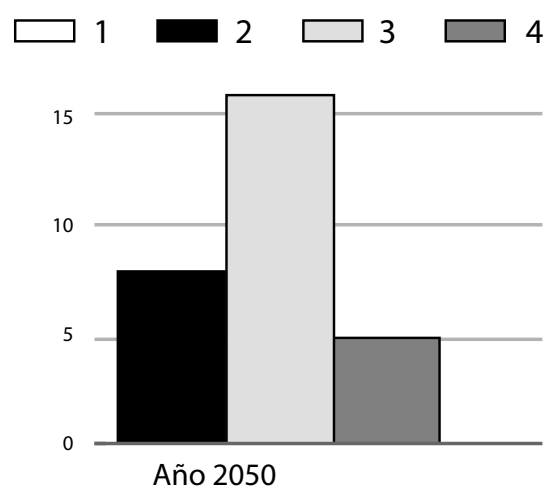

Fuente: elaboración propia.

La figura 4, indica que hay mayor tendencia a pensar que hace falta mayor preparación en el país en esta era de cambios tecnológicos a la pregunta que se hizo en materia de educación: ¿El país estará preparado para asumir los cambios en el trabajo, ocasionados por la digitalización, la Inteligencia Artificial y la Robotización?

Figura 4. Respuestas de expertos sobre los avances científicos y tecnológicos de la Cuarta Revolución Industrial

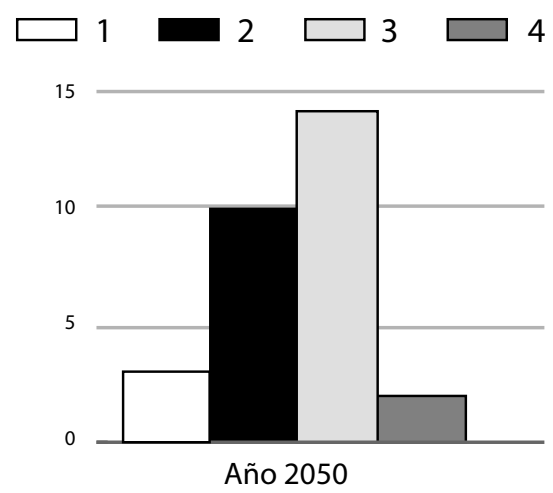

Fuente: elaboración propia.

La figura 5, muestra en consenso que los avances tecnológicos van a cambiar la forma en que se aprende y se enseña frente a la cuestión relacionada con el despliegue y adopción de las tecnologías de la Cuarta Revolución industrial, ¿La manera como se aprende y enseña, cambiaran radicalmente? 
Figura 5. Respuestas de expertos sobre el modo de enseñanza para el desarrollo de la Cuarta Revolución industrial

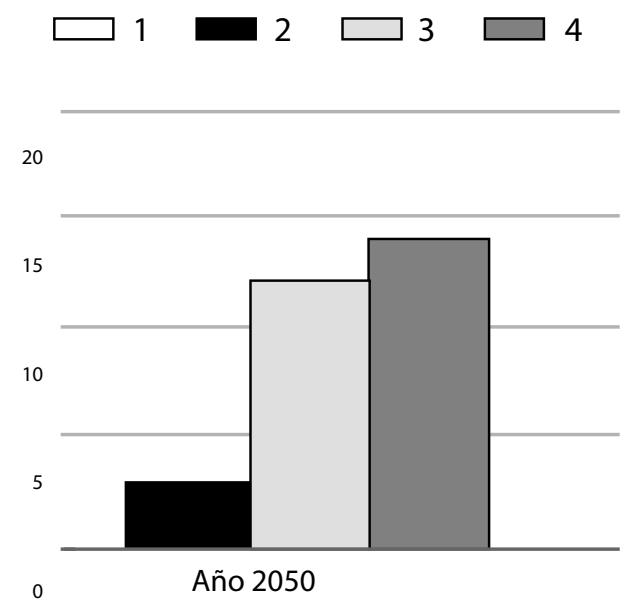

Fuente: elaboración propia.

\subsection{Taller con expertos}

Este taller se realizó el 26 de octubre de 2018 en la Sede Norte de la Universidad Central. Fue liderado por 4 expertos de diferentes áreas de especialización, los cuales realizaron sus respectivas argumentaciones y cambios respectivos según la metodología. El análisis permitió identificar los factores que fueron considerados por el grupo, como las de mayor prioridad para la educación superior en Colombia. Los expertos son:

\section{Experto 1}

Jaime Frankly Rodríguez

Vicerrector, Universidad Nacional de Colombia, Sede Bogotá.

\section{Experto 2}

Edgardo José Cuello Fuentes

Asesor en prospectiva del Ejército Nacional, Docente de posgrados del instituto Forum, Universidad de la Sabana y Docente de Tiempo Completo, Universidad de la Salle.

\section{Experto 3}

Claudia Alexandra Garzón Santos

Docente Tiempo Completo, Universidad Nacional de Colombia, área de Gestión y Organizaciones. Docente, Universidad Especialidades Espíritu Santo (ESAI). 


\section{Experto 4}

Jorge Ricardo Puentes González

Docente Hora Cátedra, Carrera de Administración de Empresas, Universidad Central.

\subsection{Pensamiento colectivo}

- ¿Cuáles de las profesiones actuales serán obsoletas en el 2050?

Los expertos plantean la necesidad de nuevos modelos de gestión de las universidades hacia una mayor profesionalización de las carreras universitarias, en donde se incluyan estas nuevas tecnologías que pueden llegar a reemplazar empleos. Mencionan que los trabajos con mayor facilidad de automatización son los que tienen tareas rutinarias, recurrentes y repetitivos, como por ejemplo los trabajos operativos.

- ¿Cómo piensan ustedes que pueden cambiar la precarización de los trabajos en Colombia al 2050?

Es una perspectiva un poco negativa, ya que los países que se adapten mejor a las tecnologías van a tener mayor ventaja. Existen posibilidades en términos de tiempo que permiten que Colombia se prepare para asumir estas tecnologías, esto se debe articular desde las mismas universidades haciendo una visión de cuáles son las tecnologías que van allegar y qué herramientas deben tener los estudiantes para usar estas tecnologías e iniciar el proceso de preparación desde ya. Este proceso también lo deben acompañar las empresas, ya que con la articulación empresa-universidad es muy posible que se llegue a una buena construcción colectiva. En términos generales, hay que apuntarle al conocimiento de una manera más drástica desde la política pública de los gobiernos, para que se pueda evitar que se amplíe la brecha tecnológica con los países desarrollados.

- ¿En Colombia vamos a una virtualización de la educación superior? ¿Cómo serán los modelos educativos para el 2050? 
En el debate se plantean las ideas sobre la pertinencia u obsolescencia de las clases magistrales y del modelo tradicional de educación, y cómo la educación virtual puede seguir siendo aprovechada, se habla de la necesidad de tener más personas educadas que puedan contribuir a la sociedad desde su área de conocimiento. Como alternativa, se apuesta por modelos mixtos de educación que concedan importancia a nuevos métodos de aprendizaje, con la incorporación de tecnologías orientadas a solucionar problemas de última generación. Ahora es necesario preparar a los estudiantes para los cambios tan rápidos que se están viviendo en el mundo, esto significa que más adelante se va a estar necesitando una formación constante en las universidades, según las necesidades que se presenten por los avances tecnológicos.

\subsection{Problemas de la Educación Superior en Colombia}

Los expertos llegaron a la conclusión de que los problemas de la educación superior en Colombia son:

- Falta de conocimiento de tecnologías

- Configuración de la educación, ya que van a desaparecer muchas carreras y solo importarán los cursos aplicados que necesite cada individuo

- Educación

- Falta de preparación tecnológica

- La competencia y aplicabilidad en conocimiento de las TIC en las aulas.

- Siempre permanecerá la desigualdad en la adquisición de conocimientos. El que los tenga y desarrolle tendrá el poder.

\subsection{Hechos portadores de futuro para la Educación Superior en Colombia}

Los expertos aportaron las siguientes reflexiones sobre cómo ven el futuro de la educación en Colombia:

- La educación siempre será la herramienta más óptima para que una sociedad supere las falencias y mejore las condiciones existenciales y materiales que le ayudan a tener una calidad de vida. En este sentido, se debe hacer una gran inversión para que los planes de estudio se modifiquen y se enfoquen en el desarrollo de competencias tecnológicas para el desarrollo de productos 
de alta innovación; de igual manera, los docentes se deben capacitar para asumir nuevos retos, donde la enseñanza tradicional se transforme para atender la nuevas demandas y el nuevo contexto educativo, dando paso al desarrollo de habilidades enmarcadas en contextos de ciencia, tecnología e innovación como modelo de desarrollo local, regional y global.

- Utilización pedagógica de los medios tecnológicos en la escuela y otros espacios.

- Teniendo en cuenta que mi tema es educación, creo que algunos de los indicadores formativos del ser humano en la educación formal y en orden de importancia serán:

- Estudiantes con una ética clara, crítica y férrea, basada en principios mínimos universales consensuados.

- Estudiantes con ciudadanías y e-ciudadanías propias de sociedades democráticas.

- Estudiantes multilingües: lectura de diferentes lenguas, códigos, patrones, etc.

- Estudiantes que sean ciudadanos del mundo, respetuosos de la diferencia, de la multiculturalidad y del otro en todos los sentidos.

- Estudiantes con una consciencia ambiental y un eco ético del cuidado del planeta que le permita actuar proactivamente en sus comportamientos públicos y privados y en sus emprendimientos laborales.

- Los procesos de cambio sugeridos a una persona, la interrelación con personas en condición de discapacidad, espacios educativos, psicología educacional y afines a la aplicación de principios de psicología social, entre otros.

- La presencia de estudiantes al aula, los medios para enseñar y la autonomía del aprendiz para la gestión de su educación.

- Sin lugar a duda, será el desarrollo de capacidades de análisis e identificación de las situaciones que se deben atender las que deben marcar el derrotero en educación.

- La enseñanza debe ser de investigación, dejar a un lado los dogmas, que ahora aparecen en línea, y buscar la atención de las necesidades de las comunidades, mirando más allá de lo obvio y pensando en potencialidades muy, muy altas. 
- Gracias a la educación se pueden crear estrategias de formación y capacitación del uso de las tecnologías.

\subsection{Escenarios de la Educación Superior en Colombia para el 2050}

- Escenario 1. Generalizar la educación digital que integre las tecnologías de la información en los procesos de aprendizaje con tecnologías de punta.

- Escenario 2. Tener políticas educativas claras que se puedan articular de forma correcta con las necesidades que tienen las empresas.

- Escenario 3. Organizar los procesos educativos superiores para que se puedan tener enfoques individuales que permitan formar profesionales, según necesidades específicas.

- Escenario 4. Desarrollo de metodologías orientadas a la creación de tecnologías de punta que ayuden a promover 1 creación de empresas en Colombia.

- Escenario 5. Programas de formación que incluyan metodologías y formación práctica en sectores de mayor demanda.

- Escenario 6. Promover y fortalecer la inclusión de más personas en el progreso educativo profesional.

- Escenario 7. Políticas presupuestales serias que permitan el desarrollo de investigación.

\section{Conclusiones}

Como resultado de esta investigación, se han logrado identificar varios factores que pueden o no afectar la educación superior en el país, ya que la incorporación de tecnologías de información significa una renovación total de los modelos educativos con los que se está trabajando actualmente. Y es que la tecnología es pieza clave en la sociedad actual, pero no es la solución mágica a los problemas educativos que se tienen y que pueden seguirse presentando en el futuro, ya que estas tecnologías no pueden cambiar por sí mismas la forma en que se aprende y se enseña, esto se debe hacer en conjunto con las empresas, el gobierno y la sociedad en general. 
En el taller con expertos, se plantearon diferentes escenarios que pueden o no afectar la educación superior en el país, algunos no son alentadores como otros, ya que no existen estrategias o factores que ayuden mitigar o aprovechar las tecnologías en la educación. Los principales retos y desafíos que tendrá la educación superior en Colombia al año 2050 por los avances tecnológicos son:

- En Colombia las tecnologías de la información y comunicación evidentemente pueden llegar a afectar en su conjunto la educación superior, pero también pueden potenciar, y facilitar los procesos de enseñanza y aprendizaje para que sea más incluyente.

- El principal consenso de los expertos se centra en la necesidad de abordar medidas urgentes, en las políticas sociales y educativas, que permitan estar preparados para asumir los cambios que traen las nuevas tecnologías, y así poder tener una lejanía del escenario más pesimista centrado en un desempleo masivo y una crisis mundial generalizada.

- Las tecnologías van a provocar una sustitución de ciertos puestos de trabajo, principalmente los que involucran tareas repetitivas u operativas, las personas con menor nivel de educación correrán un riesgo mayor por causa de la automatización y digitalización, que las personas con mayor nivel de educativo.

- Se considera que los sistemas económicos, sociales, laborales, educativos tradicionales que se conocen actualmente van a cambiar y es incierto en este momento cómo será su configuración, pero no existe un total acuerdo en que el desarrollo tecnológico vaya a generar tanta destrucción de puestos de trabajo.

- El campo de reflexión más propicio para el aporte de nuevas reflexiones sobre cambios futuros de la educación superior en Colombia, que complementen las realizadas en este estudio, se pueden centrar en las políticas, inversiones y evolución que necesita la educación superior para conducir una construcción del futuro que dé respuesta a las deudas pendientes. 


\section{Referencias}

CEPAL (2011). Prospectiva y desarrollo. Objetivos de la Prospectiva. Recuperado de https:// biblioguias.cepal.org/ProspectivayDesarrollo/Prospectiva

Colombia, Congreso de la República. Ley 30 de 1992. Por la cual se organiza el servicio público de la Educación Superior (28 diciembre 1992.)

Darcet, J. (1967). Bibliothèque de Prospective. Étapes De La Prospective. Saint German: Presses Universitaires de France.

Edgar Eslava. (2010). ¿Qué es prospectiva? Recuperado de http://www.degerencia.com/articulo/que-es-prospectiva

Fajardo, M. S. (2017). La Educación Superior Inclusiva en Algunos Países de Latinoamérica: Avances, Obstáculos y Retos. Revista Latinoamericana de Educación Inclusiva, 11(1), 171-197. https://doi.org/10.4067/S0718-73782017000100011

Fundación Telefónica. (2018). El trabajo y la revolución digital en España. Proyecto Millenium, (130). Recuperado de http://www.fundaciontelefonica.com/publicaciones

Medina Vásquez, J. (2006). Manual de prospectiva y decisión estratégica: bases teóricas e instrumentos para América Latina y el Caribe. Instituto Latinoamericano y Del Caribe de Planificación Económica y Social, (438). Recuperado de http://www.eclac.cl/ilpes/ publicaciones/xml/3/27693/manual51.pdf

Medina Vásquez, J., Becerra, S., \& Castaño, P. (2014). Prospectiva y política pública para el cambio estructural en América Latina y el Caribe. Santiago de Chile: CEPAL - Naciones Unidas.

Melo, L., Ramos, J., \& Hernández, P. (2014). La Educación Superior en Colombia: Situación Actual y Análisis de Eficiencia. Borradores de Economía, (808), 51.

Ministerio de Educación Nacional. (2009). ¿Qué es la educación superior? Recuperado de https://www.mineducacion.gov.co/1621/article-196477.html

Mojica, F. J. (2008). Dos modelos de la Escuela Voluntarista de Prospectiva Estratégica. Universidad Externado de Colombia, 1-11. Recuperado de http://www.franciscomojica. com/\#publicaciones

Núñez, M. (2018). Estrategias para 2050 en busca del mejor escenario posible. Telefónica. Recuperado de https://aunclicdelastic.blogthinkbig.com/estrategias-para-2050-en-busca-del-mejor-escenario-posible-en-espana/

Rodríguez, R. B. (2004). Las nuevas tecnologías aplicadas a la educación del siglo xxi. Revista La Tarea, 1-7. 
Suárez, M. A. C., Moreno, R. C., Camelo, O. E., Hernández, N. M., \& Velasco, J. P. (2017). Estudio prospectivo sobre la gestión del conocimiento en la educación superior en Colombia al año 2025.

Zapata, R. (2006). La escuela francesa de estrategia y prospectiva: una investigación aplicada. Revista Ciencias Estratégicas, 14(15), 55-66. 